\title{
Replica treatment of non-Hermitian disordered Hamiltonians
}

\author{
Shinsuke M. Nishigaki and Alex Kamenev \\ Department of Physics, Technion-Israel Institute of Technology, Haifa 32000, Israel
}

(September 13, 2001)

\begin{abstract}
We employ the fermionic and bosonic replicated nonlinear $\sigma$ models to treat Ginibre unitary, symplectic, and orthogonal ensembles of non-Hermitian random matrix Hamiltonians. Using saddle point approach combined with Borel resummation procedure we derive the exact large $-N$ results for microscopic density of states in all three ensembles. We also obtain tails of the density of states as well the two-point function for the unitary ensemble.

PACS numbers: $05.45+\mathrm{b}, 73.23 . \mathrm{Ps}, 75.10 . \mathrm{Nr}$
\end{abstract}

\section{INTRODUCTION}

In recent years the non-Hermitian random matrix Hamiltonians found a broad spectrum of applications in physical problems 1 . Their applicability ranges from dynamics of vortex linest and gauge theory at high baryon density to description of biological populations. On the mathematical side, the study of non-Hermitian ensembles was pioneered by Ginibre Hermitian counterparts of the Dyson ensembles and computed correlation functions for the unitary ensemble. A few decades later Mehta extended his results to the symplectic ensemble 6 . Feinberg and Zeed introduced the Hermitization trick and derived a number of results going beyond Ginibre Gaussian ensembles. The field-theoretical treatment $\mathrm{B}$ in the supersymmetric formulation $\mathrm{g}$ was introduced by Fyodorov, Sommers and Khoruzhenko 10 for the unitary ensemble. Efetov 1122 has subsequently developed the supersymmetric method for all three nonHermitian ensembles. In this paper we take an alternative route of the field-theoretical treatment, namely the replica trick 13 . This method was recently proved to be capable of describing the forrelation functions of the Hermitian Dyson ensembles14 18. So far the validity of the replica approach has been restricted to the asymptotic regions of the correlation functions only, while the supersymmetry is exact everywhere. It has, however, an important advantage of being applicable to much broader class of systems, e.g. interacting disordered systems 19 or Calogero-Sutherland model 20 .

Here we show that the replica strategy works for the Ginibre ensembles as well. Although the treatment is philosophically similar to that for the Dyson ensembles, it requires considerable technical modifications associated with the intrinsic chirality of the non-Hermitian symmetry classes. In particular, the Riemannian symmetric manifolds which are target spaces of the nonlinear $\sigma$ model differs from the corresponding Hermitian counterparts. Rather, they are subspaces of their chiral Hermitian counterparts, as may be anticipated upon Hermitization. This necessitates introduction of convenient parameterizations, calculation of the measure and finally an appropriate analytical continuation. We also extend the method by performing all order expansion around the saddle points. In the $n \rightarrow 0$ limit the resulting series may be summed up employing the Borel resummation procedure. As a result, we are able to obtain formally exact results for the density of states for all three Ginibre ensembles.

The paper is organized as follows: in Section [I] we collect analytic results known for Ginibre ensembles, and preliminaries about the replica method. In Section III we derive unitary $\sigma$ model using fermionic replica and obtain corresponding density of states, including the tails near the boundary of the spectrum support. Section IV extends the unitary $\sigma$ model to calculation of the two-point function. In Section $\mathrm{V}$ we treat the density of states for the symplectic ensemble using fermionic replica. In Section $\mathrm{VI}$ we treat the density of states for the orthogonal ensembles using bosonic replica. Finally peculiarities of the method and possible extensions are discussed in Section VII.

\section{PRELIMINARIES}

\section{A. Non-Hermitian Gaussian ensembles}

Throughout this paper we adopt Mehta's conventions and notations $\mathbf{d}$. Ginibre ensemble with $\beta=1$ (orthogonal), $\beta=2$ (unitary), or $\beta=4$ (symplectic) is defined as ensembles of $N \times N$ matrices $H$ over one of the fields: $\mathbf{R}, \mathbf{C}$, or $\mathbf{H}$. Each entry of such matrices contains $\beta$ real components which are i.i.d. variables drawn from the Gaussian distribution

$$
d \mu(H)=(\alpha \pi)^{-\beta N^{2} / 2} \mathrm{e}^{-\frac{1}{\alpha} \operatorname{tr} H H^{\dagger}} \prod_{q=1}^{\beta} \prod_{i, j=1}^{N} d H_{i j}^{(q)}
$$

$(\alpha=2,1,1$ for $\beta=1,2,4)$.

For a real $N \times N$ matrix $H(\beta=1)$, the secular equation, $\operatorname{det}(z-H)=0$, has real coefficients, so complex eigenvalues always appear in conjugate pairs. For a quaternion-real $N \times N$ matrix $H(\beta=4)$, one can find a complex $2 N \times 2 N$ matrix representation (also denoted as $H$ for notational simplicity) satisfying 


$$
\bar{H}=J H J^{-1}, \quad J=\left[\begin{array}{cc}
0 & \mathbb{1}_{N} \\
-\mathbb{1}_{N} & 0
\end{array}\right] .
$$

It implies that $\operatorname{det}(\bar{z}-H)=\overline{\operatorname{det}(z-H)}$, and thus complex eigenvalues again appear in conjugate pairs.

Spectral correlation functions are defined as

$$
R_{p}\left(z_{1}, \ldots, z_{p}\right)=\left\langle\operatorname{tr} \delta^{2}\left(z_{1}-H\right) \cdots \operatorname{tr} \delta^{2}\left(z_{p}-H\right)\right\rangle,
$$

where $\langle\cdots\rangle$ denotes averaging with $d \mu(H)$. The macroscopic density of states (DoS) of $H$ is uniform within a circle of radius $\sqrt{N}(\beta=1,2)$ or $\sqrt{2 N}(\beta=4)$. Below we collect known results for the microscopic correlation functiong-computed by the (skew-)orthogonal polynomial method 10 and by the supersymmetry method 11 .

\section{Unitary Ensemble目, 6.21}

$$
\begin{aligned}
& \pi R_{1}(z)=\frac{\mathrm{e}^{-|z|^{2}}}{\Gamma(N)} \int_{0}^{\infty} d \lambda \mathrm{e}^{-\lambda}\left(\lambda+|z|^{2}\right)^{N-1} \quad(\text { exact }), \quad(4 \mathrm{a}) \\
& \pi R_{1}(z)=1 \quad(|z| \ll \sqrt{N}), \quad=0 \quad(|z| \gg \sqrt{N}), \quad(4 \mathrm{~b}) \\
& \pi R_{1}(z)=1-\frac{\mathrm{e}^{-2 u^{2}}}{2 \sqrt{2 \pi} u} \quad(|z|=\sqrt{N}-u, 1 \ll u \ll \sqrt{N}),
\end{aligned}
$$

$$
\pi^{2} R_{2}\left(z, z^{\prime}\right)=1-\mathrm{e}^{-\left|z-z^{\prime}\right|^{2}} . \quad\left(|z|,\left|z^{\prime}\right| \ll \sqrt{N}\right)
$$

Symplectic Ensemble $[1] \quad(z=x+i y,|z| \ll \sqrt{N})$

$$
\pi R_{1}(z)=2 y^{2} \int_{0}^{1} \frac{d \lambda}{\sqrt{1-\lambda}} \mathrm{e}^{-2 y^{2} \lambda} .
$$

Orthogonal Ensemble $[1] \quad(z=x+i y,|z| \ll \sqrt{N})$

$$
\pi R_{1}(z)=2 y^{2} \int_{0}^{\infty} \frac{d \lambda}{\sqrt{1+\lambda}} \mathrm{e}^{-2 y^{2} \lambda}+\sqrt{\pi} \delta(y) .
$$

\section{B. Replica method}

In the Hermitian case, one uses the identity

$$
\begin{aligned}
\delta(x) & =\frac{1}{\pi} \operatorname{Im} \frac{1}{x-i \epsilon}=\frac{1}{\pi} \operatorname{Im} \frac{d}{d x} \log (x-i \epsilon) \\
& =\lim _{n \rightarrow 0} \frac{1}{\pi n} \operatorname{Im} \frac{d}{d x}(x-i \epsilon)^{n},
\end{aligned}
$$

which in the matrix context takes the form 13

$$
\operatorname{tr} \delta(x-H)=\lim _{n \rightarrow 0} \frac{1}{\pi n} \operatorname{Im} \frac{d}{d x} \operatorname{det}^{n}(x-i \epsilon-H) .
$$

The two distinct versions $n>0$ and $n<0$ are called fermionic and bosonic replica, correspondingly. The fermionic replicated generating functions $\left(\operatorname{det}^{n}(x-\right.$ $H$ ) averaged with $d \mu(H))$ of unitary 4 , orthogonal, symplectic15.16, and chiral unitary 17 ensembles lead to compact nonlinear $\sigma$ models on $\mathrm{U}(2 n) / \mathrm{U}(n) \times \mathrm{U}(n)$,
$\mathrm{Sp}(2 n) / \mathrm{Sp}(n) \times \mathrm{Sp}(n), \mathrm{O}(2 n) / \mathrm{O}(n) \times \mathrm{O}(n)$, and $\mathrm{U}(2 n)$, respectively, which are FF blocks of the Riemannian symmetric suferspaces of type AIII|AIII, BDI|CII, CII|BDI, and $\mathrm{A} \mid \mathrm{A}^{22}$. After appropriate parameterization of these symmetric spaces one obtains $n$-fold compact integrals, which may be evaluated in the saddle point approximation. By collecting contributions from dominant as well as subdominant saddle points, one obtains the asymptotic expressions for the correlation functions. Bosonic replica has been successfully applied to the chiral unitary ensemble 1 , see also Ref.23.

In the non-Hermitian case, one may employ the identity

$$
\delta^{2}(z)=\frac{1}{\pi} \partial_{z} \partial_{\bar{z}} \log (z \bar{z})=\lim _{n \rightarrow 0} \frac{1}{\pi n} \partial_{z} \partial_{\bar{z}}(z \bar{z})^{n},
$$

which in our context takes the form

$$
\operatorname{tr} \delta^{2}(z-H)=\lim _{n \rightarrow 0} \frac{1}{\pi n} \partial_{z} \partial_{\bar{z}} \operatorname{det}^{n}(z-H) \operatorname{det}^{n}\left(\bar{z}-H^{\dagger}\right) .
$$

This form resembles the generating function for a block off-diagonal (chiral) matrix obtained upon Hermitization 24. The difference is that the spectral parameter enters off-diagonally rather than diagonally as in the chiral case. This distinction however does not affect the resulting saddle point manifolds, which is determined at infinitesimal spectral parameters Therefore, according to Zirnbauer's celebrated result22, the saddle point manifolds are expected to be (subspaces of) $\mathrm{U}(2 n)$, $\mathrm{U}(2 n) / \mathrm{O}(2 n), \mathrm{U}(2 n) / \mathrm{Sp}(n)$ (for fermionic replica) or $\mathrm{GL}(2 n, \mathbf{C}) / \mathrm{U}(2 n), \mathrm{U}^{*}(2 n) / \mathrm{Sp}(n), \mathrm{GL}(2 n, \mathbf{R}) / \mathrm{O}(2 n)$ (for bosonic replica) for $\beta=2,4,1$, respectively, which are FF and BB blocks of the Riemannian symmetric superspaces of type A|A, AII $\mid \mathrm{AI}$, and AI $\mid \mathrm{AII}$.

Our aim is to obtain the DoS and the two-point function (cf. Eqs. (4) , (5), (6)) including exponential terms, using the replica strategy. We employ either fermionic or bosonic replica, whichever is suitable for the actual computation.

\section{GINIBRE UNITARY ENSEMBLE: DENSITY OF STATES}

Consider the fermionic replicated generating function (positive moment of the characteristic polynomial) of Ginibre unitary ensemble defined as

$$
Z_{n}(z, \bar{z})=\int_{\mathbf{C}^{N \times N}} d H \mathrm{e}^{-\operatorname{tr} H H^{\dagger}} \operatorname{det}^{n}(z-H) \operatorname{det}^{n}\left(\bar{z}-H^{\dagger}\right),
$$

where $d H=\pi^{-N^{2}} \prod_{i, j}^{N} d^{2} H^{i j}$. We denote 'color' indices by $i, j=1, \ldots, N$ and 'flavor' indices by $a, b=1, \ldots, n$. In the standard way one introduces mutually independent Grassmann variables $\psi_{a}^{i}, \bar{\psi}_{a}^{i}, \chi_{a}^{i}, \bar{\chi}_{a}^{i}$, and rewrites the generating function in terms of an auxiliary matrix $Q_{a b}$ (a well-known reexpression of the chiral unitary ensemble with flavor and color indices interchanged 25.26): 


$$
\begin{aligned}
Z_{n}(z, \bar{z}) & =\int_{\mathbf{C}^{N \times N}} d H \int d \bar{\psi} d \psi d \bar{\chi} d \chi \exp \left(-H^{i j} \bar{H}^{i j}-\bar{\psi}_{a}^{i}\left(z \delta^{i j}-H^{i j}\right) \psi_{a}^{j}-\bar{\chi}_{a}^{j}\left(\bar{z} \delta^{j i}-\bar{H}^{i j}\right) \chi_{a}^{i}\right) \\
& =\int d \bar{\psi} d \psi d \bar{\chi} d \chi \exp \left(\bar{\psi}_{a}^{i} \psi_{a}^{j} \bar{\chi}_{b}^{j} \chi_{b}^{i}-z \bar{\psi}_{a}^{i} \psi_{a}^{i}-\bar{z} \bar{\chi}_{a}^{i} \chi_{a}^{i}\right) \\
& =\int_{\mathbf{C}^{n \times n}} d Q \mathrm{e}^{-\operatorname{tr} Q Q^{\dagger}} \int d \bar{\psi} d \psi d \bar{\chi} d \chi \exp \left(-\left[\bar{\psi}_{a}^{i} \bar{\chi}_{a}^{i}\right]\left[\begin{array}{cc}
z \delta_{a b} & -Q_{a b} \\
Q_{a b}^{\dagger} & \bar{z} \delta_{a b}
\end{array}\right]\left[\begin{array}{c}
\psi_{b}^{i} \\
\chi_{b}^{i}
\end{array}\right]\right) \\
& =\int_{\mathbf{C}^{n \times n}} d Q \mathrm{e}^{-\operatorname{tr} Q Q^{\dagger}} \operatorname{det}^{N}\left[\begin{array}{cc}
z & -Q \\
Q^{\dagger} & \bar{z}
\end{array}\right]=\int_{\mathbf{C}^{n \times n}} d Q \mathrm{e}^{-\operatorname{tr} Q Q^{\dagger}} \operatorname{det}^{N}\left(z \bar{z}+Q Q^{\dagger}\right) .
\end{aligned}
$$

Here $d \psi=\prod_{a, i} d \psi_{a}^{i}$ and so forth, and $d Q=$ $\pi^{-n^{2}} \prod_{a, b}^{n} d^{2} Q_{a b}$. A complex matrix $Q \in \mathbf{C}^{n \times n}$ can be uniquely written as (singular value decomposition)

$$
\begin{aligned}
& Q=U \Lambda V, \quad U \in \mathrm{U}(n) / \mathrm{U}(1)^{n}, V \in \mathrm{U}(n), \\
& \Lambda=\operatorname{diag}\left(\lambda_{a}^{1 / 2}\right), \lambda_{a} \geq 0
\end{aligned}
$$

and a Euclidean measure $d Q$ on $\mathbf{C}^{n \times n}$ is related to normalized Haar measures $d U$ on $\mathrm{U}(n) / \mathrm{U}(1)^{n}$ and $d V$ on $\mathrm{U}(n)$ by

$$
d Q=d U d V \Delta(\lambda)^{2} \prod_{a=1}^{n} d \lambda_{a}, \quad \Delta(\lambda)=\prod_{a>b}^{n}\left(\lambda_{a}-\lambda_{b}\right) .
$$

As the integrand does not depend on $U$ and $V, Z_{n}(z, \bar{z})$ is written as an $n$-fold integral (even for finite $N$ ),

$$
Z_{n}(z, \bar{z})=\int_{0}^{\infty} \prod_{a=1}^{n}\left(d \lambda_{a} \mathrm{e}^{-\lambda_{a}}\left(\lambda_{a}+|z|^{2}\right)^{N}\right) \Delta(\lambda)^{2},
$$

up to an irrelevant constant factor that approaches unity in the replica limit. Notice the striking resemblance between the DoS (4a) and the replica generating function (13) even at finite $N$. This raises the question of whether the replica method may be applicable beyond the asymptotic analysis. We come back to this issue in Sect.VII.

\section{A. DoS in the bulk}

Now we take the large $-N$ limit. For $|z|<\sqrt{N}$, the saddle point equation

$$
1-\frac{N}{\lambda+|z|^{2}}=0
$$

has a solution $\lambda=N-|z|^{2}$. Therefore

$$
Z_{n}(z, \bar{z})=\mathrm{e}^{-n\left(N-|z|^{2}\right)} .
$$

up to an irrelevant constant given by the Selberg integral6, which goes to unity as $n \rightarrow 0$, and $\mathrm{O}\left(n^{2}\right)$ terms. On the other hand, for $|z|>\sqrt{N}$, the integral is dominated by the end point $\lambda=0$ :

$$
Z_{n}(z, \bar{z})=|z|^{2 n N} \text {. }
$$

Using relation (8), one obtains for the DoS

$$
\pi R_{1}(z)=\lim _{n \rightarrow 0} \frac{1}{n} \partial_{z} \partial_{\bar{z}} Z_{n}(z, \bar{z})=\left\{\begin{array}{ll}
1 & |z|<\sqrt{N} \\
0 & |z|>\sqrt{N}
\end{array} .\right.
$$

Therefore $N$ complex eigenvalues are uniformly distributed within a circle of radius $\sqrt{N}$, in agreement with the exact result (4b).

\section{B. DoS at the edge}

If $|z|$ is close to the edge of the circle, such that $|z|=\sqrt{N}-u$, where $1_{\sim}^{<} u \ll \sqrt{N}$, one may work out corrections to the uniform DoS. In this case both the saddle point at $\lambda_{a}=N-|z|^{2} \simeq 2 \sqrt{N} u$ and the end point $\lambda_{a}=0$ contribute to the partition function. Summing up all the contributions one finds

$$
\begin{aligned}
& Z_{n}(z, \bar{z})=\mathrm{e}^{-n\left(N-|z|^{2}\right)} \sum_{p=0}^{n}\left(\begin{array}{l}
n \\
p
\end{array}\right)(-)^{p}(2 \sqrt{N} u)^{2 p(n-p)} \mathrm{e}^{-2 p u^{2}} \\
& \int_{0}^{\infty} \prod_{a=1}^{p}\left(d \lambda_{a} \mathrm{e}^{-\frac{2 u}{\sqrt{N}} \lambda_{a}}\right) \Delta(\lambda)^{2} \times \int_{-\infty}^{\infty} \prod_{b=1}^{n-p}\left(d \lambda_{b} \mathrm{e}^{-\frac{\lambda_{b}^{2}}{2 N}}\right) \Delta(\lambda)^{2}
\end{aligned}
$$

Employing Selberg integrals and neglecting constant factors which go to unity in the $n \rightarrow 0$ limit, one obtains

$$
\begin{aligned}
& Z_{n}(z, \bar{z})= \\
& \mathrm{e}^{-n\left(N-|z|^{2}\right)} \sum_{p=0}^{n}(-)^{p} \mathrm{e}^{-2 p u^{2}}(2 u)^{2 p(n-p)-p^{2}}(2 \pi)^{\frac{n-p}{2}} D_{n}^{p},
\end{aligned}
$$

where

$$
D_{n}^{p} \equiv \prod_{a=1}^{p} \frac{\Gamma(a)^{2}}{\Gamma(n-a+1)} .
$$

Since $D_{n}^{p}=0$ for $p>n$, one may extend summation over $p$ to infinity in Eq. (19). We then perform the analytic continuation $n \rightarrow 0$. To this end we notice that $D_{n}^{p}=O\left(n^{p}\right)$ in the limit $n \rightarrow 0$. Therefore only the terms with $p=0$ and $p=1$ contribute to the partition function in the small- $n$ limit. As a result, one finds 


$$
Z_{n}(z, \bar{z})=\mathrm{e}^{-n\left(N-|z|^{2}\right)}\left(1-n \frac{\mathrm{e}^{-2 u^{2}}}{\sqrt{2 \pi}(2 u)^{3}}\right) .
$$

Employing finally Eq. (8) one obtains for the DoS close to the edge of the spectrum support

$$
\begin{aligned}
\pi R_{1}(z) & =\lim _{n \rightarrow 0} \frac{1}{n} \partial_{z} \partial_{\bar{z}} Z_{n}(z, \bar{z}) \\
& =1-\frac{\mathrm{e}^{-2 u^{2}}}{2 \sqrt{2 \pi} u}+O\left(\frac{\mathrm{e}^{-2 u^{2}}}{u^{3}}\right) .
\end{aligned}
$$

This asymptotics agree with the exact result $(4 d)$ within the validity of our approximation $1 \ll u^{2}(\ll N)$. The tail of the DoS outside the circle, $|z|>\sqrt{N}$ can not be obtained in the fermionic replica. Indeed, in this case the saddle point is situated at negative $\lambda$ and the contour of integration cannot be deformed to pass through it. As a result the integrals are dominated by the end point $\lambda=0$, which leads to the zero DoS for $|z|>\sqrt{N}$. It is possible that the bosonic replica f model may be capable to produce these tails (c.f. Ref.27).

\section{GINIBRE UNITARY ENSEMBLE: TWO-LEVEL CORRELATION}

Consider the fermionic replicated generating function with two spectral parameters,

$$
\begin{aligned}
& Z_{n}\left(z_{1}, \bar{z}_{1} ; z_{2}, \bar{z}_{2}\right)=\int_{\mathbf{C}^{N \times N}} d H \mathrm{e}^{-\operatorname{tr} H H^{\dagger}} \times \\
& \operatorname{det}^{n}\left(z_{1}-H\right) \operatorname{det}^{n}\left(\bar{z}_{1}-H^{\dagger}\right) \operatorname{det}^{n}\left(z_{2}-H\right) \operatorname{det}^{n}\left(\bar{z}_{2}-H^{\dagger}\right) .
\end{aligned}
$$

Introducing the enlarged flavor indices $A, B=1, \ldots, 2 n$ and a $2 n \times 2 n$ matrix $Z$,

$$
Z=\left[\begin{array}{cc}
z_{1} \mathbb{1}_{n} & 0 \\
0 & z_{2} \mathbb{1}_{n}
\end{array}\right]
$$

one rewrites the generating function in terms of the auxiliary matrix $Q_{A B}$ as in the previous section,

$$
Z_{n}\left(z_{1}, \bar{z}_{1} ; z_{2}, \bar{z}_{2}\right)=\int_{\mathbf{C}^{2 n \times 2 n}} d Q \mathrm{e}^{-\operatorname{tr} Q Q^{\dagger}} \operatorname{det}^{N}\left[\begin{array}{cc}
Z & -Q \\
Q^{\dagger} & \bar{Z}
\end{array}\right],
$$

with $d Q=\pi^{-4 n^{2}} \prod_{A, B}^{2 n} d^{2} Q_{A B}$.

Hereafter we concentrate on the center of the circle $\left|z_{1}\right|,\left|z_{2}\right| \ll \sqrt{N}$. At $z_{1}=z_{2}=0$, the large $-N$ saddle point equation

$$
Q^{\dagger}-N Q^{\dagger}\left(Q Q^{\dagger}\right)^{-1}=0
$$

is solved by

$$
Q=\sqrt{N} U, \quad U \in \mathrm{U}(2 n) .
$$

For $z_{1}, z_{2}$ finite and of order unity, one can write for such $Q$

$$
\begin{aligned}
& \operatorname{det}\left[\begin{array}{cc}
Z & -Q \\
Q^{\dagger} & \bar{Z}
\end{array}\right] \simeq \exp \left(-\frac{1}{2 N} \operatorname{tr}\left[\begin{array}{cc}
0 & -U^{-1} \bar{Z} \\
U Z & 0
\end{array}\right]^{2}\right) \\
& =\exp \left(\frac{1}{N}\left(2 n|z|^{2}+\left|\frac{\omega}{2}\right|^{2} \operatorname{tr} U s U^{-1} s\right)\right),
\end{aligned}
$$

where

$$
z=\frac{z_{1}+z_{2}}{2}, \quad \omega=z_{1}-z_{2}, \quad s=\left[\begin{array}{cc}
\mathbb{1}_{n} & 0 \\
0 & -\mathbb{1}_{n}
\end{array}\right] .
$$

Therefore,

$$
\begin{aligned}
& Z_{n}\left(z_{1}, \bar{z}_{1} ; z_{2}, \bar{z}_{2}\right)= \\
& \mathrm{e}^{-2 n\left(N-|z|^{2}\right)} \int_{\mathrm{U}(2 n)} d U \exp \left(\frac{|\omega|^{2}}{4} \operatorname{tr} U s U^{-1} s\right),
\end{aligned}
$$

up to an irrelevant constant factor that approaches unity in the replica limit. Here $d U$ is a Haar measure on $\mathrm{U}(2 n)$. The above integrand is invariant under

$$
U \rightarrow U\left[\begin{array}{cc}
u & 0 \\
0 & u^{\prime}
\end{array}\right], \quad u, u^{\prime} \in \mathrm{U}(n)
$$

so the saddle point manifold shrinks down to $\mathrm{U}(2 n) / \mathrm{U}(n) \times \mathrm{U}(n)$. Adopting the parameterization of this coset manifold employed by Verbaarschot and Zirnbauere8

$U s U^{-1}=\left[\begin{array}{ll}u & 0 \\ 0 & v\end{array}\right]\left[\begin{array}{cc}\cos \boldsymbol{\Theta} & \sin \boldsymbol{\Theta} \mathrm{e}^{i \boldsymbol{\Phi}} \\ \sin \boldsymbol{\Theta} \mathrm{e}^{-i \boldsymbol{\Phi}} & -\cos \boldsymbol{\Theta}\end{array}\right]\left[\begin{array}{cc}u^{-1} & 0 \\ 0 & v^{-1}\end{array}\right]$,

$\boldsymbol{\Theta}=\operatorname{diag}\left(\theta_{a}\right), \mathbf{\Phi}=\operatorname{diag}\left(\phi_{a}\right), \quad u, v \in \mathrm{U}(n) / \mathrm{U}(1)^{n}$,

$d U=d u d v \prod_{a=1}^{n} d \phi_{a} \prod_{a=1}^{n} d \cos \theta_{a} \Delta(\cos \theta)^{2}$,

and substituting

$$
\operatorname{tr} U s U^{-1} s=2 \operatorname{tr} \cos \Theta=2 \sum_{a=1}^{n} \cos \theta_{a},
$$

one obtains

$$
\begin{aligned}
& Z_{n}\left(z_{1}, \bar{z}_{1} ; z_{2}, \bar{z}_{2}\right)= \\
& \mathrm{e}^{-2 n\left(N-|z|^{2}\right)} \int_{-1}^{1} \prod_{a=1}^{n}\left(d \lambda_{a} \mathrm{e}^{\frac{|\omega|^{2}}{2} \lambda_{a}}\right) \Delta(\lambda)^{2},
\end{aligned}
$$

where $\lambda_{a}=\cos \theta_{a}$.

The replica limit of the above $n$-fold integral has been discussed extensively by many authors 28. 16. 12,20 (see also Ref.2 for its asymptotic analysis). The integral (28) over Grassmann manifold $\mathrm{U}(2 n) / \mathrm{U}(n) \times \mathrm{U}(n)$ with a height function $\operatorname{tr} U s U^{-1} s$ satisfies the criteria of the Duistermaat-Heckman localization theorem 30.31 and thus the saddle point method applied to Eq. (28) is exact for an arbitrary value of $|\omega|^{2}$. For positive large 
$t=|\omega|^{2} / 2$, the integral (32) may be 'approximated' by contributions from the end points $\lambda_{a}= \pm 1$,

$$
\begin{aligned}
& \int_{-1}^{1} \prod_{a=1}^{n}\left(d \lambda_{a} \mathrm{e}^{t \lambda_{a}}\right) \Delta(\lambda)^{2} \\
& =\sum_{p=0}^{n}(-)^{p}\left(F_{n}^{p}(1)\right)^{2} \frac{\mathrm{e}^{(n-2 p) t}}{(2 t)^{(n-p)^{2}+p^{2}}},
\end{aligned}
$$

where

$$
F_{n}^{p}(k) \equiv\left(\begin{array}{l}
n \\
p
\end{array}\right) \prod_{a=1}^{p} \frac{\Gamma(1+a / k)}{\Gamma(1+(n-a+1) / k)}
$$

for $p>0$ and $F_{n}^{0}(k)=1$. One may extend summation over $p$ to infinity, since the binomial coefficient $\left(\begin{array}{l}n \\ p\end{array}\right) \equiv 0$ for $p>n$. After this one may perform analytical continuation, employing the small- $n$ limit,

$$
F_{n}^{p}(k)=n \frac{(-1)^{p+1}}{p} \prod_{a=1}^{p} \frac{\Gamma(1+a / k)}{\Gamma(1-(a-1) / k)}+\mathrm{O}\left(n^{2}\right) .
$$

Accordingly the integral reads, for small $n$,

$$
\int_{-1}^{1} \prod_{a=1}^{n}\left(d \lambda_{a} \mathrm{e}^{t \lambda_{a}}\right) \Delta(\lambda)^{2} \simeq \frac{\mathrm{e}^{n t}}{(2 t)^{n^{2}}}-n^{2} \frac{\mathrm{e}^{(n-2) t}}{(2 t)^{n^{2}-2 n+2}} .
$$

Using this formula, one obtains

$$
\begin{aligned}
& Z_{n}\left(z_{1}, \bar{z}_{1} ; z_{2}, \bar{z}_{2}\right) \simeq \\
& \mathrm{e}^{-2 n\left(N-|z|^{2}\right)}\left(\frac{\mathrm{e}^{n|\omega|^{2} / 2}}{|\omega|^{2 n^{2}}}-n^{2} \frac{\mathrm{e}^{(n-2)|\omega|^{2} / 2}}{|\omega|^{2\left(n^{2}-2 n+2\right)}}\right) .
\end{aligned}
$$

The two-point function is given by

$$
\begin{aligned}
\pi^{2} R_{2}\left(z_{1}, z_{2}\right) & =\lim _{n \rightarrow 0} \frac{1}{n^{2}} \partial_{z_{1}} \partial_{\bar{z}_{1}} \partial_{z_{2}} \partial_{\bar{z}_{2}} Z_{n}\left(z_{1}, \bar{z}_{1} ; z_{2}, \bar{z}_{2}\right) \\
& =1-\mathrm{e}^{-\left|z_{1}-z_{2}\right|^{2}}+O\left(\frac{\mathrm{e}^{-\left|z_{1}-z_{2}\right|^{2}}}{\left|z_{1}-z_{2}\right|^{2}}\right) .
\end{aligned}
$$

This agrees with the exact result $(4 \mathrm{~d})$ within the validity of approximation $1 \ll\left|z_{1}-z_{2}\right|^{2}(\ll N)$. The disconnected part $1=\pi^{2} R_{1}\left(z_{1}\right) R_{1}\left(z_{2}\right)$ is given by the replica symmetric saddle point $p=0$, whereas the connected part $-\mathrm{e}^{-\left|z_{1}-z_{2}\right|^{2}}$ is given by the replica nonsymmetric saddle point, $p=1$. In the latter case, the $(n-1)$-fold integral around $\lambda=1$ and a one-fold integral around $\lambda=-1$ 'interact' through the Van der Monde determinant. Taking this interaction into account in the perturbative manner, one finds that all terms proportional to $\mathrm{e}^{-\left|z_{1}-z_{2}\right|^{2}} /\left|z_{1}-z_{2}\right|^{2 k}(k \geq 1)$ are canceled. We have checked this statement explicitly to the order $k=1$. In general it is a manifestation of the Duistermaat-Heckman localization theorem 31 .

\section{GINIBRE SYMPLECTIC ENSEMBLE}

For a $2 N \times 2 N$ complex matrix representation of an $N \times N$ quaternion-real matrix $H$, the constraint (2) may be solved by

$$
H=\left[\begin{array}{cc}
S & T \\
-\bar{T} & \bar{S}
\end{array}\right], \quad S, T \in \mathbf{C}^{N \times N} .
$$

The fermionic replicated generating function of Ginibre symplectic ensemble thus reads

$$
\begin{aligned}
Z_{n}(z, \bar{z}) & =\int_{\mathbf{H}^{N \times N}} d H \mathrm{e}^{-\frac{1}{2} \operatorname{tr} H H^{\dagger}} \operatorname{det}^{n}(z-H) \operatorname{det}^{n}(\bar{z}-H) \\
& =\int_{\mathbf{C}^{N \times N}} d S d T \mathrm{e}^{-\operatorname{tr} S S^{\dagger}-\operatorname{tr} T T^{\dagger}} \operatorname{det}^{n}\left(z-\left[\begin{array}{cc}
S & T \\
-\bar{T} & \bar{S}
\end{array}\right]\right) \operatorname{det}^{n}\left(\bar{z}-\left[\begin{array}{cc}
S & T \\
-\bar{T} & \bar{S}
\end{array}\right]\right),
\end{aligned}
$$

where $d H=d S d T=\pi^{-2 N^{2}} \prod_{i, j}^{N} d^{2} S^{i j} d^{2} T^{i j}$. Once again we introduce enlarged flavor indices $A, B=1, \ldots, 2 n$ and express the generating function in terms of the auxiliary matrix $Q_{A B}$,

$$
\begin{aligned}
& Z_{n}(z, \bar{z})=\int_{\mathbf{C}^{N \times N}} d S d T \int d \bar{\psi} d \psi d \bar{\chi} d \chi \exp \left(-S^{i j} \bar{S}^{i j}-T^{i j} \bar{T}^{i j}-\left[\bar{\psi}_{A}^{i} \bar{\chi}_{A}^{i}\right]\left(\left[\begin{array}{cc}
\delta^{i j} & 0 \\
0 & \delta^{i j}
\end{array}\right] Z_{A B}-\left[\begin{array}{cc}
S^{i j} & T^{i j} \\
-\bar{T}^{i j} & \bar{S}^{i j}
\end{array}\right] \delta_{A B}\right)\left[\begin{array}{c}
\psi_{B}^{j} \\
\chi_{B}^{j}
\end{array}\right]\right) \\
& =\int d \bar{\psi} d \psi d \bar{\chi} d \chi \exp \left(-\bar{\psi}_{A}^{i} Z_{A B} \psi_{B}^{i}-\bar{\chi}_{A}^{i} Z_{A B} \chi_{B}^{i}-\psi_{A}^{i} \bar{\psi}_{A}^{j} \bar{\chi}_{B}^{j} \chi_{B}^{i}+\chi_{A}^{i} \bar{\psi}_{A}^{j} \bar{\chi}_{B}^{j} \psi_{B}^{i}\right) \\
& =\int_{\mathcal{M}} d Q \int d \bar{\psi} d \psi d \bar{\chi} d \chi \\
& \exp \left(-\frac{1}{2} Q_{A B} \bar{Q}_{A B}+\frac{1}{2} Q_{A B}\left(\bar{\psi}_{A}^{i} \bar{\chi}_{B}^{i}+\bar{\psi}_{B}^{i} \bar{\chi}_{A}^{i}\right)+\frac{1}{2} \bar{Q}_{B A}\left(\chi_{A}^{j} \psi_{B}^{j}+\chi_{B}^{j} \psi_{A}^{j}\right)-\bar{\psi}_{A}^{i} Z_{A B} \psi_{B}^{i}-\bar{\chi}_{A}^{i} Z_{A B} \chi_{B}^{i}\right) \\
& =\int_{\mathcal{M}} d Q \mathrm{e}^{-\frac{1}{2} \operatorname{tr} Q Q^{\dagger}} \int d \bar{\psi} d \psi d \bar{\chi} d \chi \exp \left(-\left[\bar{\psi}_{A}^{i} \chi_{A}^{i}\right]\left[\begin{array}{cc}
Q_{A B} & -Z_{A B} \\
Z_{A B} & Q_{A B}^{\dagger}
\end{array}\right]\left[\begin{array}{c}
\bar{\chi}_{B}^{i} \\
\psi_{B}^{i}
\end{array}\right]\right)=\int_{\mathcal{M}} d Q \mathrm{e}^{-\frac{1}{2} \operatorname{tr} Q Q^{\dagger}} \operatorname{det}^{N}\left[\begin{array}{cc}
Q & -Z \\
Z & Q^{\dagger}
\end{array}\right],
\end{aligned}
$$


where $z=x+i y, Z=x \mathbb{1}_{2 n}+i y s$. Here $d Q=$ $(2 \pi)^{-2 n^{2}-n} \prod_{A>B}^{2 n} d^{2} Q_{A B}$, and the integration domain $\mathcal{M}$ is a set of $2 n \times 2 n$ complex symmetric matrices, because its antisymmetric part decouples from the fermionic bilinear. A similar procedure was adopted for the chiral symplectic ensemble 32 .

Hereafter we concentrate on the center of the circle $|z| \ll \sqrt{2 N}$. The large $-N$ saddle point equation

$$
\frac{1}{2} Q^{\dagger}-N Q^{\dagger}\left(Q Q^{\dagger}\right)^{-1}=0
$$

is solved by

$$
Q=\sqrt{2 N} U, \quad U \in \mathrm{U}(2 n)
$$

For $z$ finite and of order unity, one can write for such $Q$,

$$
\begin{aligned}
& \operatorname{det}\left[\begin{array}{cc}
Q & -Z \\
Z & Q^{\dagger}
\end{array}\right] \simeq \exp \left(-\frac{1}{4 N} \operatorname{tr}\left[\begin{array}{cc}
0 & -U^{-1} Z \\
U Z & 0
\end{array}\right]^{2}\right) \\
& =\exp \left(\frac{1}{N}\left(n x^{2}-\frac{y^{2}}{2} \operatorname{tr} U s U^{-1} s\right)\right)
\end{aligned}
$$

Therefore,

$$
Z_{n}(z, \bar{z})=\mathrm{e}^{-n\left(2 N-x^{2}\right)} \int_{\substack{\mathrm{U}(2 n) \\ U=U^{T}}} d U \exp \left(-\frac{y^{2}}{2} \operatorname{tr} U s U^{-1} s\right)
$$

up to an irrelevant constant factor that approaches unity in the replica limit. Here $d U$ is a Haar measure on a group quotient $\mathrm{U}(2 n) / \mathrm{O}(2 n)$ that is isomorphic to the space of $2 n \times 2 n$ unitary symmetric matrices through a canonical projection $g \mapsto U=g g^{T}$. Since the integrand is invariant under

$$
U \rightarrow\left[\begin{array}{cc}
u & 0 \\
0 & u^{\prime}
\end{array}\right] U\left[\begin{array}{cc}
u^{T} & 0 \\
0 & u^{\prime T}
\end{array}\right], \quad u, u^{\prime} \in \mathrm{U}(n)
$$

the saddle point manifold shrinks down to the intersection of $\mathrm{U}(2 n) / \mathrm{O}(2 n)$ and $\mathrm{U}(2 n) / \mathrm{U}(n) \times \mathrm{U}(n)$. Following Zirnbauer and Haldane 33 , one can parameterize the matrix $U$ and the measure $d U$ as

$$
\begin{aligned}
& U= {\left[\begin{array}{ll}
u & 0 \\
0 & v
\end{array}\right]\left[\begin{array}{cc}
\cos \boldsymbol{\Theta} & i \sin \boldsymbol{\Theta} \\
i \sin \boldsymbol{\Theta} & \cos \boldsymbol{\Theta}
\end{array}\right]\left[\begin{array}{cc}
u^{T} & 0 \\
0 & v^{T}
\end{array}\right], } \\
& \boldsymbol{\Theta}=\operatorname{diag}\left(\theta_{a}\right), u, v \in \mathrm{U}(n) \\
& d U=d u d v \prod_{a=1}^{n} d \sin \theta_{a}\left|\Delta\left(\cos ^{2} \theta\right)\right|
\end{aligned}
$$

Computation of the above Jacobian is detailed in Appendix A. By substituting

$$
\operatorname{tr} U s U^{-1} s=4 \sum_{a=1}^{n} \cos ^{2} \theta_{a}-2 n,
$$

we obtain the generating function in the form of the $n$ fold integral
$Z_{n}(z, \bar{z})=\mathrm{e}^{-n\left(2 N-|z|^{2}\right)} \int_{0}^{1} \prod_{a=1}^{n}\left(\frac{d \lambda_{a}}{\sqrt{1-\lambda_{a}}} \mathrm{e}^{-2 y^{2} \lambda_{a}}\right)|\Delta(\lambda)|$

where $\lambda_{a}=\cos ^{2} \theta_{a}$. Again we have encountered a striking resemblance between the DoS (5) and the generating function (50).

For $y^{2} \gg 1$ this integral is dominated by vicinities of the two end points. By considering $t \equiv 2 y^{2}$ to have a negative imaginary part (possibly infinitesimal), one may deform the integration contour to the upper half plane as $[0,1] \rightarrow[0, i \infty)+(1+i \infty, 1]$. Employing Selberg integrals, one may evaluate all the contributions with $p$ integrals along $[1,1+i \infty)$ taken in the vicinity of $\lambda=1$, whereas the remaining $n-p$ integrals along $[0, i \infty)$ coming from the vicinity of $\lambda=0$. Whenever the end point $\lambda_{a}=0$ is adopted for some $a$, one may safely expand $\left(1-\lambda_{a}\right)^{-1 / 2}$ in $\lambda_{a}$, while it ought to be treated exactly when the other end point $\lambda_{a}=1$ is chosen. Meanwhile we approximate $\left(1-\lambda_{a}\right)^{-1 / 2} \simeq 1$ for the former case and find

$$
\begin{aligned}
I_{n}(t) & =\int_{0}^{1} \prod_{a=1}^{n}\left(\frac{d \lambda_{a}}{\sqrt{1-\lambda_{a}}} \mathrm{e}^{-t \lambda_{a}}\right)|\Delta(\lambda)| \\
& \simeq \sum_{p=0}^{n}\left(F_{n}^{p}(2)\right)^{2} \frac{\Gamma\left(\frac{1+n-p}{2}\right)}{\Gamma\left(\frac{1}{2}\right)} \frac{(-1)^{\frac{p^{2}}{2}} \mathrm{e}^{-p t}}{t^{\frac{(n-p)(n-p+1)}{2}+\frac{p^{2}}{2}}}
\end{aligned}
$$

As in previous sections, one may extend summation over $p$ to infinity and perform analytical continuation, employing the small- $n$ limit (35). Only $p=0$ and $p=1$ terms contribute in the replica limit $n \rightarrow 0$, since $p \geq 2$ terms are of the order $\mathrm{O}\left(n^{2}\right)$. As a result,

$$
I_{n}(t) \simeq t^{-n / 2}+n \frac{i \sqrt{\pi}}{2} t^{-1 / 2} \mathrm{e}^{-t}
$$

In order to obtain perturbative (i.e. powers of $1 / t$ ) corrections, one expand the $\left(1-\lambda_{a}\right)^{-1 / 2}$ factor in powers of $\lambda_{a}$ for the replica-symmetric $(p=0)$ term,

$$
\begin{aligned}
& \int_{0}^{i \infty} \prod_{a=1}^{n}\left(d \lambda_{a} \mathrm{e}^{-t \lambda_{a}}\right)|\Delta(\lambda)| \prod_{b=1}^{n}\left(1-\lambda_{b}\right)^{-1 / 2} \\
= & \int_{0}^{i \infty} \prod_{a=1}^{n}\left(d \lambda_{a} \mathrm{e}^{-t \lambda_{a}}\right)|\Delta(\lambda)| \exp \left(\sum_{k=1}^{\infty} \frac{\omega_{k}}{2 k}\right),
\end{aligned}
$$

where $\omega_{k} \equiv \sum_{a=1}^{n} \lambda_{a}^{k}$ are elemantary symmetric pqlynomials. In Appendix B we prove by loop equations 34 that the RHS of Eq. (53) is equal to 


$$
\begin{aligned}
& \int_{0}^{i \infty} \prod_{a=1}^{n}\left(d \lambda_{a} \mathrm{e}^{-t \lambda_{a}}\right)|\Delta(\lambda)| \exp \left(\sum_{k=1}^{\infty} \frac{\omega_{1}^{k}}{2 k}\right) \\
= & \int_{0}^{i \infty} \prod_{a=1}^{n}\left(d \lambda_{a} \mathrm{e}^{-t \lambda_{a}}\right)|\Delta(\lambda)|\left(1-\sum_{b=1}^{n} \lambda_{b}\right)^{-1 / 2} \\
= & \left(1+\partial_{t}\right)^{-1 / 2} \int_{0}^{i \infty} \prod_{a=1}^{n}\left(d \lambda_{a} \mathrm{e}^{-t \lambda_{a}}\right)|\Delta(\lambda)|
\end{aligned}
$$

plus terms of order $O\left(n^{2}\right)$. Here $\left(1+\partial_{t}\right)^{-1 / 2}$ stands for a formal series

$$
\left(1+\partial_{t}\right)^{-1 / 2}=\sum_{k=0}^{\infty} \frac{(2 k-1) ! !}{(-2)^{k} k !} \frac{d^{k}}{d t^{k}}
$$

Accordingly the generating function reads

$$
\begin{aligned}
Z_{n}(z, \bar{z}) & =\mathrm{e}^{-n\left(2 N-|z|^{2}\right)} I_{n}\left(2 y^{2}\right), \\
I_{n}(t) & =\left(1+\partial_{t}\right)^{-1 / 2} t^{-n / 2}+n \frac{i \sqrt{\pi}}{2} t^{-1 / 2} \mathrm{e}^{-t} .
\end{aligned}
$$

The DoS is then given by

$$
\begin{aligned}
\pi R_{1}(z) & =\lim _{n \rightarrow 0} \frac{1}{n} \partial_{z} \partial_{\bar{z}} Z_{n}(z, \bar{z}) \\
= & 1-\frac{1}{2}\left\{\partial_{t}, t\right\} \partial_{t}\left(1+\partial_{t}\right)^{-1 / 2} \log t+i \sqrt{\pi t} \mathrm{e}^{-t}
\end{aligned}
$$

$t=2 y^{2}$, up to terms of order $O\left(t^{-1 / 2} \mathrm{e}^{-t}\right)$. On the other hand, one can evaluate the asymptotic expansion of the exact result (5) for $\operatorname{Re} y^{2} \gg 1$ and $\operatorname{Im} y^{2}<0$ by using the same saddle point method (it is formally equivalent to the calculation given above for $n=1$ ),

$$
\begin{aligned}
\pi R_{1}(z) & =t\left(\int_{0}^{i \infty} \frac{d \lambda}{\sqrt{1-\lambda}} \mathrm{e}^{-t \lambda}-\mathrm{e}^{-t} \int_{0}^{i \infty} \frac{d \lambda^{\prime}}{\sqrt{-\lambda^{\prime}}} \mathrm{e}^{-t \lambda^{\prime}}\right) \\
& =t\left(1+\partial_{t}\right)^{-1 / 2} t^{-1}+i \sqrt{\pi t} \mathrm{e}^{-t} .
\end{aligned}
$$

One may confirm the equality of Eqs. (57) and (58) either by using a canonical commutation relation $\left[\partial_{t}, t\right]=1$ to bring e.g. all $t$ 's to the right of $\partial_{t}$ 's, or by explicit substitution of Eq. (55) to derive an identical asymptotic series

$$
\pi R_{1}(z)=\sum_{k=0}^{\infty} \frac{(2 k-1) ! !}{(2 y)^{2 k}}+i \sqrt{2 \pi} y \mathrm{e}^{-2 y^{2}} .
$$

The formal Borel resummation of the series, Eq. (59), leads the exact result for the DoS, Eq. (5). Therefore our fermionic replica method gives correctly all orders of perturbative terms as well as the nonperturbative term of the DoS. Although the imaginary terms $\pm i \sqrt{2 \pi} y \mathrm{e}^{-2 y^{2}}$ are artifacts of locating $y^{2}$ in the positive side of the lower or upper half plane and are obviously absent for real positive $y^{2}$ (i.e. the positive real axis is the Stokes line for the asymptotic expansions), it is their presence and coincidence that enables us to identify the two expressions: (50) with a Laplacian applied and (5), viewed as complex functions of $y^{2}$.

\section{GINIBRE ORTHOGONAL ENSEMBLE}

For a reason that will be mentioned below, we consider the bosonic replicated generating function (negative moment of the characteristic polynomial) for the case of Ginibre orthogonal ensemble:

$$
\begin{aligned}
& Z_{-n}(z, \bar{z})= \\
& \int_{\mathbf{R}^{N \times N}} d H \mathrm{e}^{-\frac{1}{2} \operatorname{tr} H H^{T}} \operatorname{det}^{-n}(z-H) \operatorname{det}^{-n}(\bar{z}-H),
\end{aligned}
$$

$d H=(2 \pi)^{-N^{2}} \prod_{i, j}^{N} d H^{i j}$. Again we introduce enlarged flavor indices $A, B=1, \ldots, 2 n$ and a $2 n \times 2 n$ matrix $Z$. To calculate a negative power of the determinant (60) with the help of commuting variables $\bar{\phi}, \phi$, one has to ensure convergence bychoosing $\operatorname{Im} z>0$ and inserting a matrix $\sqrt{s}$ defined as 35

$$
\sqrt{s}=\left[\begin{array}{cc}
\mathbb{1}_{n} & 0 \\
0 & i \mathbb{1}_{n}
\end{array}\right]
$$

as a result:

$$
\begin{aligned}
& Z_{-n}(z, \bar{z})=\int_{\mathbf{R}^{N \times N}} d H \int_{\mathbf{C}^{2 n \times N}} d \bar{\phi} d \phi \exp \left(-\frac{1}{2} H^{i j} H^{i j}+i \bar{\phi}_{A}^{i} \sqrt{s}_{A B}\left(Z_{B C} \delta^{i j}-\delta_{B C} H^{i j}\right) \sqrt{s}_{C D} \phi_{D}^{j}\right) \\
& =\int_{\mathbf{C}^{2 n \times N}} d \bar{\phi} d \phi \exp \left(-\frac{1}{2} \bar{\phi}_{A}^{i} s_{A B} \phi_{B}^{j} \bar{\phi}_{C}^{i} s_{C D} \phi_{D}^{j}+i \bar{\phi}_{A}^{i} \sqrt{s}_{A B} Z_{B C} \sqrt{s}_{C D} \phi_{D}^{i}\right) \\
& =\int_{\mathcal{M}} d Q d \tilde{Q} \mathrm{e}^{-\frac{1}{2} \operatorname{tr} Q \tilde{Q}} \int_{\mathbf{C}^{2 n \times N}} d \bar{\phi} d \phi \exp \left(\frac{i}{2}\left[\bar{\phi}_{A}^{i} \phi_{A}^{i}\right] \sqrt{s}_{A B}\left[\begin{array}{cc}
Q_{B C} & Z_{B C} \\
Z_{B C} & \tilde{Q}_{B C}
\end{array}\right] \sqrt{s}_{C D}\left[\begin{array}{c}
\bar{\phi}_{D}^{i} \\
\phi_{D}^{i}
\end{array}\right]\right) \\
& =\int_{\mathcal{M}} d Q d \tilde{Q} \mathrm{e}^{-\frac{1}{2} \operatorname{tr} Q \tilde{Q}} \operatorname{det}^{-\frac{N}{2}}\left[\begin{array}{ll}
Q & Z \\
Z & \tilde{Q}
\end{array}\right] \text {. }
\end{aligned}
$$


Here $d \bar{\phi} d \phi=(2 \pi)^{-2 N n} \prod_{A, i} d^{2} \phi_{A}^{i}, \quad d Q d \tilde{Q}=$ $(2 \pi)^{-2 n^{2}-n} \prod_{A \geq B}^{2 n} d^{2} Q_{A B} d^{2} \tilde{Q}_{A B}$, and the integration domain $\mathcal{M}$ is a set of pairs of $2 n \times 2 n$ complex symmetric matrices $(Q, \tilde{Q})$ that are mutually Hermitian conjugated, $\tilde{Q}=Q^{\dagger}$. The restriction to symmetric matrices is because their antisymmetric parts decouple from the bosonic bilinears.

Hereafter we concentrate on the center of the circle, $|z| \ll \sqrt{N}$. The large $-N$ saddle point equations

$$
\frac{1}{2} \tilde{Q}+\frac{N}{2} \tilde{Q}(Q \tilde{Q})^{-1}=0, \quad \frac{1}{2} Q+\frac{N}{2}(Q \tilde{Q})^{-1} Q=0
$$

imply

$$
Q \tilde{Q}=-N \mathbb{1}_{2 n} .
$$

Clearly this saddle point manifold lies outside $\mathcal{M}$, for $Q \tilde{Q}$ would have to be positive definite. For this saddle point to be admissible, one must deform the original integration domain $\mathcal{M}=\left\{Q_{A B}^{+} \in \mathbf{R}, Q_{A B}^{-} \in i \mathbf{R}\right\}$, $Q^{ \pm} \equiv(Q \pm \tilde{Q}) / 2$, into $\widetilde{\mathcal{M}}=\left\{Q_{A B}^{+} \in \mathbf{R}, Q_{A B}^{-} \in \mathcal{C}_{A B}\right\}$, where the contour $\mathcal{C}_{A B}$ is shown in Figure 1.

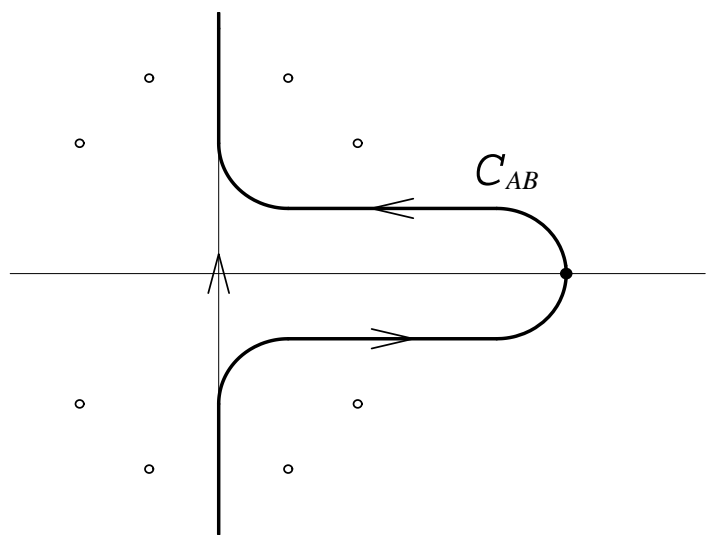

FIG. 1. Schematic view of the integration contours in the complex $Q_{A B}^{-}$plane. The original contour (imaginary axis) can be deformed to $\mathcal{C}_{A B}$ that passes through the saddle point $(\bullet)$ without encircling the poles (o) of the determinant, each of which appears to the left of the saddle point and symmetrically in four quadrants.

The width of the deformed section of the contour scales as $1 / \sqrt{N}$. Therefore, as long as $y \gg 1 / \sqrt{N} \rightarrow 0$, the poles of $\operatorname{det}^{-N / 2}\left[\begin{array}{ll}Q & Z \\ Z & \tilde{Q}\end{array}\right]$ are away from the deformed contour. Then the saddle point equations can be solved by

$$
\begin{aligned}
& Q=\sqrt{N} U, \quad \tilde{Q}=-\sqrt{N} U^{-1}, \\
& U \in \operatorname{GL}(2 n, \mathbf{R}), \quad U=U^{T} .
\end{aligned}
$$

For $z$ small and finite of order unity, one can write for such $Q$ and $\tilde{Q}$,

$$
\begin{aligned}
& \operatorname{det}\left[\begin{array}{cc}
Q & Z \\
Z & \tilde{Q}
\end{array}\right] \simeq \exp \left(-\frac{1}{2 N} \operatorname{tr}\left[\begin{array}{cc}
0 & U^{-1} Z \\
-U Z & 0
\end{array}\right]^{2}\right) \\
& =\exp \left(\frac{1}{N}\left(2 n x^{2}-y^{2} \operatorname{tr} U s U^{-1} s\right)\right),
\end{aligned}
$$

where $z=x+i y, Z=x \mathbb{1}_{2 n}+i y s$.

After having deformed the contour of the integration over $(Q, \tilde{Q})$, one must re-ensure convergence of the $\phi$ integrals in Eq. (61) by keeping the real part of the quadratic form

$$
\begin{aligned}
& \frac{i}{2}[\bar{\phi} \phi] \sqrt{s}\left[\begin{array}{cc}
Q & Z \\
Z & \tilde{Q}
\end{array}\right] \sqrt{s}\left[\begin{array}{c}
\bar{\phi} \\
\phi
\end{array}\right] \\
= & -y \sum_{A=1}^{2 n}\left|\phi_{A}\right|^{2}+i x \sum_{a=1}^{n}\left(\left|\phi_{a}\right|^{2}-\left|\phi_{a+n}\right|^{2}\right) \\
& +[\operatorname{Re} \phi \operatorname{Im} \phi] \sqrt{s}\left[\begin{array}{rr}
i Q^{+} & Q^{-} \\
Q^{-} & -i Q^{+}
\end{array}\right] \sqrt{s}\left[\begin{array}{c}
\operatorname{Re} \phi \\
\operatorname{Im} \phi
\end{array}\right],
\end{aligned}
$$

to be negative definite. A sufficient condition is

$$
\begin{aligned}
& \left(\sqrt{s} Q^{+} \sqrt{s}\right)_{A B}, \quad i\left(\sqrt{s} Q^{-} \sqrt{s}\right)_{A B} \in \mathbf{R} \\
& \Leftrightarrow s Q^{ \pm} s= \pm Q^{ \pm} \Leftrightarrow Q s=s \tilde{Q} .
\end{aligned}
$$

This condition is actually necessary for the real part of the quadratic form to be negative definite for arbitrarily small $y>0$. At the saddle point $Q=-\tilde{Q}^{-1} \equiv \sqrt{N} U$, it implies a constraint $U s U s=-\mathbb{1}$. Therefore,

$$
\begin{aligned}
& Z_{-n}(z, \bar{z})=\mathrm{e}^{n\left(N-x^{2}\right)} \int_{\mathcal{D}} d U \exp \left(\frac{y^{2}}{2} \operatorname{tr} U s U^{-1} s\right), \\
& \mathcal{D}=\left\{U \in \operatorname{GL}(2 n, \mathbf{R}) \mid U=U^{T},(U s)^{2}=-\mathbb{1}\right\},
\end{aligned}
$$

up to an irrelevant constant factor that approaches unity in the replica limit. Here $d U$ is a Haar measure on the symmetric space of symmetric $\mathrm{GL}(2 n, \mathbf{R})$ matrices, consisting of $2 n+1$ disconnected components with $2 n-k$ positive and $k$ negative eigenvalues. The group quotient $\mathrm{GL}(2 n, \mathbf{R}) / \mathrm{O}(2 n)$, which is the saddle point manifold of the bosonic replicated $\sigma$ model for the chiral Gaussian orthogonal ensemble, is isomorphic to the $k=0$ component by a canonical projection $g \mapsto U=g g^{T}$, but the component used here as the integration domain $\mathcal{D}$ is a subspace of the $k=n$ component. This difference between non-Hermitian and chiral Hermitian ensembles is not surprising, because the symmetry breaking parameter $z$ plays a critical rôle as a regularization parameter, either in the bosonic replica method or in the bosonic part of the supersymmetry method in general.

Eq. (68) is invariant under

$$
U \rightarrow\left[\begin{array}{cc}
u & 0 \\
0 & u^{\prime}
\end{array}\right] U\left[\begin{array}{cc}
u^{T} & 0 \\
0 & u^{\prime T}
\end{array}\right], \quad u, u^{\prime} \in \mathrm{O}(n),
$$

so the saddle point manifold shrinks down to the intersection of $\mathcal{D}$ and $\mathrm{GL}(2 n, \mathbf{R}) / \mathrm{O}(n) \times \mathrm{O}(n)$. A generic solution to the constraint (69) is given by

$$
\begin{gathered}
U=\left[\begin{array}{ll}
u & 0 \\
0 & v
\end{array}\right]\left[\begin{array}{ll}
\sinh \boldsymbol{\Theta} & \cosh \boldsymbol{\Theta} \\
\cosh \boldsymbol{\Theta} & \sinh \boldsymbol{\Theta}
\end{array}\right]\left[\begin{array}{cc}
u^{T} & 0 \\
0 & v^{T}
\end{array}\right], \\
\boldsymbol{\Theta}=\operatorname{diag}\left(\theta_{a}\right), u, v \in \mathrm{O}(n) .
\end{gathered}
$$


Comparing Eq. (71) with (47), the Haar measure $d U$ in terms of this parametrization is formally obtained from Eq. (48) by a replacement $\boldsymbol{\Theta} \rightarrow \pi / 2-i \boldsymbol{\Theta}$. Accordingly,

$$
d U=d u d v \prod_{a=1}^{n} d \cosh \theta_{a}\left|\Delta\left(\sinh ^{2} \theta\right)\right| .
$$

By substituting

$$
\operatorname{tr} U s U^{-1} s=-\operatorname{tr} U^{2}=-4 \sum_{a=1}^{n} \sinh ^{2} \theta_{a}-2 n,
$$

one finally obtains

$$
Z_{-n}(z, \bar{z})=\mathrm{e}^{n\left(N-|z|^{2}\right)} \int_{0}^{\infty} \prod_{a=1}^{n}\left(\frac{d \lambda_{a}}{\sqrt{1+\lambda_{a}}} \mathrm{e}^{-2 y^{2} \lambda_{a}}\right)|\Delta(\lambda)|,
$$

where $\lambda_{a}=\sinh ^{2} \theta_{a}$. Note that an identical expression also follows from the case with $y=\operatorname{Im} z<0$. Once again we have encountered the resemblance between the DoS (6) and the generating function (74).

For $y^{2} \gg 1 / N$ this integral is dominated by the vicinity of the end point $\lambda_{a}=0$. By temporarily approximating $\left(1+\lambda_{a}\right)^{-1 / 2} \simeq 1$, one obtains

$$
Z_{-n}(z, \bar{z}) \simeq \mathrm{e}^{n\left(N-|z|^{2}\right)} t^{-n / 2}, \quad t=2 y^{2}>0 .
$$

In order to obtain the perturbative corrections in $1 / t$, one expands the $\left(1+\lambda_{a}\right)^{-1 / 2}$ factor in powers of $\lambda_{a}$ as in Sect. V. Accordingly the generating function reads

$$
Z_{-n}(z, \bar{z})=\mathrm{e}^{n\left(N-|z|^{2}\right)}\left(1-\partial_{t}\right)^{-1 / 2} t^{-n / 2} \text {. }
$$

The DoS is then given by

$$
\begin{aligned}
\pi R_{1}(z) & =\lim _{n \rightarrow 0} \frac{1}{-n} \partial_{z} \partial_{\bar{z}} Z_{-n}(z, \bar{z}) \\
& =1+\frac{1}{2}\left\{\partial_{t}, t\right\} \partial_{t}\left(1-\partial_{t}\right)^{-1 / 2} \log t
\end{aligned}
$$

On the other hand, one can evaluate the asymptotic expansion of the smooth part of the exact result (6) for $\operatorname{Re} y^{2} \gg 1$ (this calculation is formally equivalent to the above given one for $n=1$ ),

$$
\pi R_{1}(z)=t\left(1-\partial_{t}\right)^{-1 / 2} t^{-1} \quad(t>0) .
$$

One can confirm the equality of Eqs. (77) and (78) either by using $\left[\partial_{t}, t\right]=1$ or by explicitly deriving an identical asymptotic series

$$
\pi R_{1}(z)=\sum_{k=0}^{\infty} \frac{(-1)^{k}(2 k-1) ! !}{(2 y)^{2 k}}
$$

The formal Borel resummation of this series leads to the smooth part of the exact result (6). The $\delta$ function peak of the DoS localized at $y=0$ cannot directly be captured by our bosonic replica treatment that requires a finite imaginary part of 236 . However it can be restored from the Borel-reconstructed smooth part through a normalization sum rule

$$
\int_{-\infty}^{\infty}\left(\pi R_{1}(y)-1\right) d y=0
$$

with a correct coefficient $\sqrt{\pi}$. Therefore the bosonic replica method correctly gives all orders of the perturbative terms as well as the absence of exponentially decaying terms (and the Stokes line at the positive real $y^{2}$ axis), and indirectly the $\delta$ function term of the DoS.

Finally we comment on the fermionic replica treatment of the orthogonal ensemble. Following the same steps as in Sect. V, one may obtain Eq. (45) with the integration domain being $2 n \times 2 n$ antisymmetric unitary matrices, which is isomorphic to a group quotient $\mathrm{U}(2 n) / \operatorname{Sp}(n)$ by a canonical projection $g \mapsto U=g J g^{T}$ (accompanied by a trivial change $2 N \rightarrow N)$. At present we are not aware of an appropriate parameterization, such as Eqs. (47) or (71), of the intersection of this coset and $\mathrm{U}(2 n) / \mathrm{U}(n) \times \mathrm{U}(n)$. Consequently these generating functions are not available in the form of $n$-fold integrals as in Eqs. (13), (32), (50), and (74) that are suitable for the asymptotic analyses. This difficulty also arises in the bosonic replica treatment of the symplectic ensemble, whose saddle point manifold is an intersection of $\mathrm{U}^{*}(2 n) / \operatorname{Sp}(n)$ and $\mathrm{U}^{*}(2 n) / \mathrm{U}^{*}(n) \times \mathrm{U}^{*}(n)$. It is currently not clear to us whether this difficulty is merely a technical obstacle or has an essential reason behind it.

\section{DISCUSSIONS}

We have derived the fermionic or bosonic replicated nonlinear $\sigma$ models for Ginibre unitary, symplectic, and orthogonal ensembles. The corresponding symmetric manifolds are subspaces of $\mathrm{U}(2 n), \mathrm{U}(2 n) / \mathrm{O}(2 n)$, and of a space adjacent to $\mathrm{GL}(2 n, \mathbf{R}) / \mathrm{O}(2 n)$ correspondingly, in conformity with Zirnbauer's classification. The proper parameterization of the manifolds and subsequent integration of irrelevant degrees of freedom lefts one with the $n$-fold compact or noncompact angular integral. Performing the asymptotic analysis of such integral, one obtains sums over variety of possible saddle points both replica-symmetric and replica nonsymmetric. After analytical continuation, $n \rightarrow 0$, only replica-symmetric contribution and possibly few nonsymmetric one survive. The former are responsible for the perturbative (polynomial in inverse large parameter), while the latter for the nonperturbative (essentially singular) contributions to the correlation functions. For the DoS calculation, it is possible to evaluate the infinite order perturbative expansions near these saddle points. One may also show that the Borel resummation of such expansions after $n \rightarrow 0$ 
limit leads to the exact results valid for arbitrary spectral parameter.

We recapitulate our findings for the non-Hermitian ensembles at hand. The bulk of the DoS in the unitary case (constant) is fully given by the replica-symmetric saddle point. The nonsymmetric contributions show up only if one approaches the very edge of the spectral support, giving the exponentially small deviations from the abrupt behavior on the border of the circle. The irreducible part of the two-point correlation function (exponentially decaying with the distance between the eigenvalues) is fully given by a single replica nonsymmetric saddle point. In the symplectic case the situation is different. In addition to the symmetric contribution that is determined to all polynomial orders, the nonsymmetric contribution exists already on the level of the bulk DoS. It brings an exponentially small correction (not oscillatory as in the case of Dyson ensembles) to the symmetric contribution, which is crucial in making contact with the exact result. In the orthogonal case, there only exists a single replica-symmetric saddle point that gives all polynomial orders of the DoS, and the exponential corrections to the symmetric contribution are absent. One can restore the $\delta$ functional peak from the smooth part of DoS reconstructed by the Borel resummation. The presence/absence of exponential corrections is a direct consequence of compact/noncompactness of the saddle point manifold which is the target space of the fermionic/bosonic replicated nonlinear $\sigma$ models.

Finally we list possible lines of future investigation:

- The resemblance generally observed between replicated generating functions (13), (50), (74) and corresponding $\operatorname{DoS}(4 \mathrm{a}),(5),(6)$, as well as a surprising small- $n$ reduction (B9) of the formers to essentially one-component integrals, makes us speculate that the replica treatment may be applicable to Ginibre ensembles beyond Borel resummation or asymptotic analyses (for large symmetry breaking parameters $|\omega|, y$ or at large $N$ ) and could indeed produce full exact results. Presumably it could be done by applying a complete set of loop equations to the $n$-fold integrals to reduce them directly to one-fold integrals37.

- Application to off-diagonal parts of the remainipg two Altland-Zirnbauer superconducting chiral classes 38 , i.e. non-Hermitian complex symmetric and complex selfdual matrices 24 , should be interesting in its own right, as analytic results have not yet been obtained via other methods. It would require appropriate parameterization of the FF or BB blocks of the Riemannian symmetric superspaces $\mathrm{D} \mid \mathrm{C}$ and $\mathrm{C} \mid \mathrm{D}$.

- Starting from Hatano-Nelson disorderedHamiltonian, either with strong or weak non-Hermiticity 10.11 , one may obtain a finite-dimensional nonlinear $\sigma$ model of the corresponding symmetry class. Our formulation and results should serve as a ground upon which non-Hermitian disordered Hamiltonians in the diffusive regime (with or without interactions) may be treated.

\section{ACKNOWLEDGMENTS}

We are indebted to A. Altland and M. Zirnbauer for valuable advises and conversations. S. M. N. thanks M. Moshe for warm hospitality at Technion. This work was supported in part (S. M. N.) by the Israel Science Foundation (ISF) and (A. K.) by the BSF-9800338 grant.

\section{APPENDIX A: JACOBIAN}

In this Appendix we compute a Jacobian asspciated with the parameterization (47) following Ref.33, Sect. VIIF and App. A., where it was proven that

(Zirnbauer-Haldane): Let $G$ be a Lie group, $G_{e}$ a subgroup that commutes with $s, K$ another subgroup whose right action on $G$ leaves a function $f$ of $G$ invariant, $A$ a maximal Abelian subgroup for the Cartan decomposition of $G$ with respect to $K, M$ a subgroup of $G_{e} \cap K$ that commutes with all elements of $A$, and $A^{+}$an open subset of $A$ such that the map $\phi:\left(G_{e} / M\right) \times A^{+} \rightarrow$ $G / K,(g M, a) \mapsto g a K$ is bijective. Let $d g$ be a Haar measure on $G, d g_{K}$ a Haar measure on $G / K$, and $d a$ a Euclidean measure on $A$. Let $T(\bullet)$ denote a Lie algebra for a Lie group and a tangent space at the origin for a group quotient. $T(G)=T(G / K) \oplus T(K)$ decomposes into the even $(e)$ and odd $(o)$ elements with respect to an involuntary automorphism $g \mapsto s g s$. As the action of $\operatorname{ad}(\log a)$, $a \in A^{+}$, maps $T(K)_{e}+T(G / K)_{o} \rightarrow T(G / K)_{o}+T(K)_{e}$ and $T(K)_{o}+T(G / K)_{e} \rightarrow T(G / K)_{e}+T(K)_{o}$, the set of positive roots $\Delta^{+}$of $G$ decomposes into $\Delta_{e}^{+}$and $\Delta_{o}^{+}$ accordingly. Then the Jacobian $J(a)$ associated with the $\operatorname{map} \phi$,

$$
\int_{G / K} f(g K) d g_{K}=\int_{A^{+}}\left(\int_{G_{e}} f(g a K) d g\right) J(a) d a
$$

is given by

$$
J(a)=\prod_{\alpha \in \Delta_{e}^{+}} \sinh \alpha(\log a) \prod_{\alpha \in \Delta_{o}^{+}} \cosh \alpha(\log a) .
$$

In the case of fermionic replica for Ginibre symplectic ensemble, we take

$$
\begin{aligned}
& G=\mathrm{U}(2 n), K=\mathrm{O}(2 n), G_{e}=\mathrm{U}(n) \times \mathrm{U}(n), \\
& A \ni a=\exp \left(\frac{i}{2}\left[\begin{array}{cc}
0 & \boldsymbol{\Theta} \\
\boldsymbol{\Theta} & 0
\end{array}\right]\right)=\left[\begin{array}{cc}
\cos (\boldsymbol{\Theta} / 2) & i \sin (\boldsymbol{\Theta} / 2) \\
i \sin (\boldsymbol{\Theta} / 2) & \cos (\boldsymbol{\Theta} / 2)
\end{array}\right], \\
& \boldsymbol{\Theta}=\operatorname{diag}\left(\theta_{a}\right), d a=\prod_{a=1}^{n} d \theta_{a}, \\
& f(g K)=\exp \left(-\frac{y^{2}}{2} \operatorname{tr} g g^{T} s\left(g g^{T}\right)^{-1} s\right),
\end{aligned}
$$

and identify $g g^{T}$ in Eq. (A4) with $U$ in Eq. 45. For $g \in G_{e}$ 


$$
\begin{aligned}
& \operatorname{tr}(g a)(g a)^{T} s\left((g a)(g a)^{T}\right)^{-1} s=\operatorname{tr} a^{2} s a^{-2} s \\
= & \operatorname{tr}\left[\begin{array}{cc}
\cos \boldsymbol{\Theta} & i \sin \boldsymbol{\Theta} \\
i \sin \boldsymbol{\Theta} & \cos \boldsymbol{\Theta}
\end{array}\right]\left[\begin{array}{cc}
\mathbb{1} & 0 \\
0 & -\mathbb{1}
\end{array}\right]\left[\begin{array}{cc}
\cos \boldsymbol{\Theta} & -i \sin \boldsymbol{\Theta} \\
-i \sin \boldsymbol{\Theta} & \cos \boldsymbol{\Theta}
\end{array}\right]\left[\begin{array}{cc}
\mathbb{1} & 0 \\
0 & -\mathbb{1}
\end{array}\right]=2 \sum_{a=1}^{n}\left(\cos ^{2} \theta_{a}-\sin ^{2} \theta_{a}\right),
\end{aligned}
$$

and thus the integration over $G_{e}$ is trivial. Without loss of generality one may restrict $\{\theta\}$ in a cell $\theta_{1} \geq \theta_{2} \geq \cdots \geq$ $\theta_{n} \geq 0$. The eigensystem of $\operatorname{ad}(\log a) X \equiv[\log a, X]=\alpha(\log a) X$ is given by

\section{Positive Root}

$$
\begin{aligned}
& \alpha=i \theta_{a} \quad \text { Eigenvector } \\
& \alpha=i \frac{\theta_{a} \pm \theta_{b}}{2} \in\left\{\begin{array}{l}
\Delta_{o}^{+} \\
\Delta_{e}^{+}
\end{array} \quad(1 \leq a<b \leq n) \quad X_{A B}=\left\{\begin{array}{l}
\delta_{A a}^{(+)} \delta_{B b}^{(\mp)}+\delta_{A b}^{( \pm)} \delta_{B a}^{(-)} \in T(G / K)_{e}+T(K)_{o} \\
\delta_{A a}^{(+)} \delta_{B b}^{(+)}-\delta_{A b}^{( \pm)} \delta_{B a}^{(-)} \in T(G / K)_{o}+T(K)_{e}
\end{array},\right.\right.
\end{aligned}
$$

where $\delta_{A a}^{( \pm)} \equiv \delta_{A a} \pm \delta_{A a+n}$. Accordingly,

$$
\begin{aligned}
J(a) d a & =\prod_{a=1}^{n} d \theta_{a} \prod_{a=1}^{n} \cos \theta_{a} \prod_{a<b}^{n}\left(\cos \frac{\theta_{a}-\theta_{b}}{2} \cos \frac{\theta_{a}+\theta_{b}}{2} \sin \frac{\theta_{a}-\theta_{b}}{2} \sin \frac{\theta_{a}+\theta_{b}}{2}\right) \\
& =\prod_{a=1}^{n} d \sin \theta_{a} \prod_{a>b}^{n}\left(\cos ^{2} \theta_{a}-\cos ^{2} \theta_{b}\right)=\prod_{a=1}^{n} d \sin \theta_{a} \Delta\left(\cos ^{2} \theta\right),
\end{aligned}
$$

up to an irrelevant positive constant. In a generic cell, one would have

$$
J(a) d a=\prod_{a=1}^{n} d \sin \theta_{a}\left|\Delta\left(\cos ^{2} \theta\right)\right| .
$$

\section{APPENDIX B: LOOP EQUATION}

In this Appendix we derive loop equation 34 for an (auxiliary) Laguerre orthogonal ensemble and solve them in the replica limit, as it is essential in deriving asymptotic series for the generating functions 17 .

Consider a set of $n$ random non-negative numbers $\left\{\lambda_{a}\right\}$ whose unnormalized joint probability distribution is given by

$$
d \rho(\lambda)=\prod_{a=1}^{n} d \lambda_{a} \mathrm{e}^{-t \lambda_{a}}|\Delta(\lambda)| .
$$

Let $\langle\cdots\rangle$ denote an average with respect to $d \rho(\lambda)$. An expectation value of a product of elemantary symmetric polynomials $\omega_{p}=\sum_{a} \lambda_{a}^{p}$,

$$
\left\langle\prod_{i=1}^{s} \omega_{p_{i}}\right\rangle, \quad p_{i} \in \mathbf{N},
$$

is called a loop amplitude. Loop equations (a.k.a. Virasoro constraints 39 ) are derived from integrals of total derivatives

$$
\int_{0}^{\infty} \prod_{a=1}^{n} d \lambda_{a} \sum_{b=1}^{n} \frac{\partial}{\partial \lambda_{b}}\left(\lambda_{b}^{p_{0}+1} \prod_{i=1}^{s} \omega_{p_{i}} \prod_{c=1}^{n} \mathrm{e}^{-t \lambda_{c}}|\Delta(\lambda)|\right)=0
$$

$\left(p_{i}=0,1, \ldots\right)$. By applying derivatives to each of the factors, one obtains

$$
\begin{aligned}
& \frac{p_{0}+1}{2}\left\langle\omega_{p_{0}} \prod_{i=1}^{s} \omega_{p_{i}}\right\rangle+\frac{1}{2} \sum_{p=0}^{p_{0}}\left\langle\omega_{p_{0}-p} \omega_{p} \prod_{i=1}^{s} \omega_{p_{i}}\right\rangle \\
& +\sum_{i=1}^{s} p_{i}\left\langle\omega_{p_{0}+p_{i}} \prod_{j(\neq i)}^{s} \omega_{p_{j}}\right\rangle=t\left\langle\omega_{p_{0}+1} \prod_{i=1}^{s} \omega_{p_{i}}\right\rangle .
\end{aligned}
$$

Starting from $\omega_{0}=n$, this set of loop equations recursively determines whole loop amplitudes. First few of them are

$$
\begin{aligned}
& \left\langle\omega_{1}\right\rangle=\frac{n(1+n)}{2 t}, \\
& \left\langle\omega_{2}\right\rangle=\frac{n\left(1+2 n+n^{2}\right)}{2 t^{2}}, \\
& \left\langle\omega_{1}^{2}\right\rangle=\frac{n\left(2+3 n+2 n^{2}+n^{3}\right)}{4 t^{2}}, \\
& \left\langle\omega_{3}\right\rangle=\frac{n\left(8+19 n+16 n^{2}+5 n^{3}\right)}{8 t^{3}}, \\
& \left\langle\omega_{1} \omega_{2}\right\rangle=\frac{n\left(4+9 n+7 n^{2}+3 n^{3}+n^{4}\right)}{4 t^{3}}, \\
& \left\langle\omega_{1}^{3}\right\rangle=\frac{n\left(8+14 n+13 n^{2}+9 n^{3}+3 n^{4}+n^{5}\right)}{8 t^{3}}, \ldots
\end{aligned}
$$

Degree-counting in Eq. (B3) leads to

$$
\left\langle\prod_{i=1}^{s} \omega_{p_{i}}\right\rangle=t^{-\sum_{i=1}^{s} p_{i}}\left(v_{p_{1}, \ldots, p_{s}} n+O\left(n^{2}\right)\right)
$$


for $p_{i}=1,2, \ldots$.

Now we prove a surprisingly simple formula

$$
v_{p_{1}, \ldots, p_{s}}=\frac{1}{2}\left(\left(\sum_{i=1}^{s} p_{i}\right)-1\right) !, \quad p_{i}=1,2, \ldots,
$$

by induction: Eq. (B6) holds at degree 1, $v_{1}=1 / 2$. Assume that it holds for all loop amplitudes of total degrees $\sum_{i=0}^{s} p_{i}=k$. An amplitude of the degree $k+1$, $v_{p_{0}+1, p_{1}, \ldots, p_{s}}$ with $p_{0}+1, p_{1}, \ldots, p_{s} \geq 1$ is given as a linear combination of these amplitudes by the loop equation (B3). If $p_{0} \geq 1$, its LHS gives

$$
\begin{aligned}
v_{p_{0}+1, p_{1}, \ldots, p_{s}} & =\left(\frac{p_{0}+1}{2}+\frac{p_{0}-1}{2}+\sum_{i=1}^{s} p_{i}\right) \frac{1}{2}\left(\sum_{i=0}^{s} p_{i}-1\right) ! \\
& =\frac{1}{2}\left(\sum_{i=0}^{s} p_{i}\right) !
\end{aligned}
$$

thus Eq. (B6) also holds for $v_{p_{0}+1, p_{1}}, \ldots, p_{s}$. If $p_{0}=0$, the first two terms of the LHS of Eq. (B3) are of higher orders in $n$, so one again has

$$
v_{1, p_{1}, \ldots, p_{s}}=\left(\sum_{i=1}^{s} p_{i}\right) \frac{1}{2}\left(\sum_{i=1}^{s} p_{i}-1\right) !=\frac{1}{2}\left(\sum_{i=1}^{s} p_{i}\right) ! .
$$

Therefore Eq. (B6) holds for any loop amplitude of the total degree $k+1$. End of proof. It means that the loop amplitude depends only on the total degree of the symmetric polynomials in the small- $n$ (replica) limit 40 ,

$$
\left\langle\prod_{i=1}^{s} \omega_{p_{i}}\right\rangle=\left\langle\omega_{1}^{\sum_{i=1}^{s} p_{i}}\right\rangle(1+O(n))
$$

As long as $\operatorname{Re} t>0$ and $\operatorname{Im} t<0$, one can rotate the integration contours of $\lambda$ 's from $[0, \infty)$ to $[0, i \infty)$ without ever modifying the loop equations and violating convergence. This justifies the transition from Eq. (53) to 54.

* email: shinsuke, kamenev@physics.technion.ac.il

${ }^{1}$ See e.g. Y. V. Fyodorov, in: Supersymmetry and Trace Formulae: Chaos and Disorder, eds. I. Lerner et al., Kluwer Academic/Plenum Pub. (New York, 1999) p.293, for further references.

${ }^{2}$ N. Hatano and D. R. Nelson, Phys. Rev. Lett. 77, 570 (1996).

${ }^{3}$ M. A. Stephanov, Phys. Rev. Lett. 76, 4472 (1996).

${ }^{4}$ D. R. Nelson and N. M. Shnerb, Phys. Rev. E 58, 1383 (1998).

${ }^{5}$ J. Ginibre, J. Math. Phys. 6, 440 (1965).

${ }^{6}$ M. L. Mehta, Random Matrices, 2nd Ed., Academic Press (San Diego, 1991).
7 J. Feinberg and A. Zee, Nucl. Phys. B 504, 579 (1997); ibid. 501, 643 (1997); cond-mat/0104072.

${ }^{8}$ F. J. Wegner, Z. Phys. B 35, 207 (1979).

${ }^{9}$ K. B. Efetov, Adv. Phys. 32, 53 (1983); Supersymmetry in Disorder and Chaos, Cambridge Univ. Press (Cambridge, 1997).

${ }^{10}$ Y. V. Fyodorov, B. A. Khoruzhenko, and H.-J. Sommers, Phys. Lett. A 226, 46 (1997);

Y. V. Fyodorov, H.-J. Sommers, and B. A. Khoruzhenko, Ann. Inst. Henri Poincaré 68, 449 (1998).

${ }^{11}$ K. B. Efetov, Phys. Rev. Lett. 79, 491 (1997); Phys. Rev. B 56, 9630 (1997).

12 A. V. Kolesnikov and K. B. Efetov, Phys. Rev. Lett. 84, 5600 (2000).

13 S. F. Edwards and P. W. Anderson, J. Phys. F 5, 89 (1975).

14 A. Kamenev and M. Mézard, J. Phys. A 32, 4373 (1999).

15 A. Kamenev and M. Mézard, Phys. Rev. B 60, 3944 (1999).

${ }^{16}$ I. V. Yurkevich and I. V. Lerner, Phys. Rev. B 60, 3955 (1999).

17 D. Dalmazi and J. J. M. Verbaarschot, Nucl. Phys. B 592, 419 (2001).

${ }^{18}$ G. Akemann, P. H. Damgaard, D. Dalmazi, and J. J. M. Verbaarschot, Nucl. Phys. B 601, 77 (2001).

19 A. M. Finkel'stein, Zh. Eksp. Teor. Fiz. 84, 168 (1983) [Sov. Phys. JETP 57, 97 (1983)].

${ }^{20}$ D. M. Gangardt and A. Kamenev, cond-mat/0102405.

21 The upper bound estimated in Eq. (15.1.35) of Ref.8, which was shown to converge to $\mathrm{e}^{-u^{2}} /(2 \sqrt{\pi} u)$, is not saturated in a region of the parameter $1 \ll u^{2} \ll N$. Applying the saddle point approximation as employed in Sect. IIIB, to the integral representation of the finite- $N$ result (4a), one obtains the correct asymptotics (4c).

${ }^{22}$ M. R. Zirnbauer, J. Phys. A 29, 7113 (1996).

${ }^{23}$ Y. V. Fyodorov, math-ph/0106006.

${ }^{24}$ M. B. Hastings, cond-mat/9909234.

${ }^{25}$ E. V. Shuryak and J. J. M. Verbaarschot, Nucl. Phys. A 560, 306 (1993).

${ }^{26}$ A. V. Andreev, B. D. Simons, and N. Taniguchi, Nucl. Phys. B 432, 487 (1994).

27 A. Cavagna, I. Giardina, and G. Parisi, Phys. Rev. B 57, 11251 (1998).

28 J. J. M. Verbaarschot and M. R. Zirnbauer, J. Phys. A 18, 1093 (1985).

${ }^{29}$ P. J. Forrester, Phys. Lett. A 179, 127 (1993).

${ }^{30}$ J. J. Duistermaat and G. Heckman, Invent. Math. 69, 259 (1982).

${ }^{31}$ M. R. Zirnbauer, cond-mat/9903338.

${ }^{32}$ M. A. Halasz and J. J. M. Verbaarschot, Phys. Rev. D 52, 2563 (1995).

${ }^{33}$ M. R. Zirnbauer and F. D. M. Haldane, Phys. Rev. B 52, 8729 (1995).

34 A. A. Migdal, Phys. Rept. 102, 199 (1983).

${ }^{35}$ L. Schäfer and F. Wegner, Z. Phys. B 38, 113 (1980).

${ }^{36}$ In the $y \rightarrow 0$ limit, complex conjugate pairs of poles in Fig. 1 merge at the real axis, so the integration domain $\mathcal{M}$ cannot be deformed to $\widetilde{\mathcal{M}}$ without picking up the residues. This is the origin of the $\delta(y)$ function peak of the DoS (6).

${ }^{37}$ Note that in this paper we have not utilized a loop equation at $p_{0}=-1$ (the $L_{-1}$ constraint), which relates an $n$-fold integral to an $(n-1)$-fold integral arising from the lower 
end of the partial integration.

${ }^{38}$ A. Altland and M. R. Zirnbauer, Phys. Rev. Lett. 76, 3420 (1996).

${ }^{39}$ M. Fukuma, H. Kawai, and R. Nakayama, Int. J. Mod. Phys. A 6, 1385 (1991).
40 This result is a peculiarity of an orthogonal ensemble and does not hold for auxiliary ensembles with pther powers of the Vandermonde determinant. See e.g. Ref.47, Eq. (94) for a counterexample. 